\title{
MODULE STRUCTURE ON INVARIANT JACOBIANS
}

\author{
NANHUA XI
}

\begin{abstract}
In this paper we show that Yau's conjecture om highest weights of invariant Jacobians holds for arbitrary connected semisimple algebraic groups over an algebraically closed field of characteristic 0 .
\end{abstract}

\section{Introduction}

This work was motivated by a conjecture of Stephen Yau on the set of highest weights of invariant Jacobians by an $\operatorname{sl}(2, \mathbf{C})$ action, which was proposed in 1985, see 2.2 for a precise statement. The conjecture arose from Yau's study of singularities, see $[2,5-7]$.

It was well known that Brieskorn in his talk of ICM1970 established a relation between simple Lie algebras and simple singularities. There is no further generalization to arbitrary singularities. In 1981, Mather and Yau proved that the complex structure of the isolated hypersurface singularity defined by an analytic function $f$ determines and is determined by its moduli algebra $A(f)$ which is $\mathbb{C}\left[\left[x_{1}, \ldots, x_{n}\right]\right] /\left(f, \frac{\partial f}{\partial x_{1}}, \ldots\right.$, $\left.\frac{\partial f}{\partial x_{n}}\right)$, see [2]. The algebra $A(f)$ is a finite-dimensional commutative algebras. Yau defined the Lie algebra $L(f)$ to be the derivation algebra of $A(f)$, which are often called Yau algebra now. In the process of proving that $L(f)$ is a finite-dimensional solvable Lie algebra, Yau made his conjecture nearly 30 years ago. A special case of Yau's conjecture was proved in [4] and was applied to prove Yau algebras are solvable (see [9]). Yau's work establishes a connection between the theory of isolated singularities and the theory of solvable Lie algebras. In particular, one can construct deformation of solvable Lie algebras by studying deformation of singularities (see for example [3]).

Yau's conjecture was proved for the following cases: (a) $n \leq 5$, see [8], (b) irreducible action, see [4], (c) some special cases for $n=6$,8; see [10,11]. Kempf did some interesting work related to Yau's conjecture (see [1]), which we will recall in the next section.

In this paper, we will establish several module homomorphisms; see Theorem 3.1 and Theorem 3.3. Combining a result of Kempf, a consequence of one module homomorphism is that Yau's conjecture on highest weights of invariant Jacobians holds for arbitrary connected semisimple algebraic groups over an algebraically closed field of characteristic 0; see Corollary 3.2 and remark to it. We will also give a direct proof for Yau's conjecture in Section 4.2.

Received by the editors May 6, 2012.

1991 Mathematics Subject Classification. 20C99, 17B10.

Key words and phrases. Jacobian, module structure, group, Lie algebra. 


\section{Notations}

2.1. Let $G$ be either a group or a Lie algebra, $K$ a field and $\rho$ a representation of $G$ on $V=K^{n}$. Then $G$ acts on the $\operatorname{ring} A=K\left[x_{1}, x_{2}, \ldots, x_{n}\right]$ of polynomial functions on $K^{n}$ and on the ring $R=K\left(x_{1}, x_{2}, \ldots, x_{n}\right)$ of rational functions on $K^{n}$, by abuse of notation, the two representations are denoted by the same letter $\tau$. The subspace $A_{d}$ of $A$ consisting of homogenous polynomials of degree $d$ is a $G$-submodule of $A$. For a rational function $f$ in $R$, the Jacobian $J(f)$ is the subspace $R$ spanned by all the partial derivatives $\frac{\partial f}{\partial x_{i}}$, the $r$ th Jacobian $J_{r}(f)$ is the subspace spanned by all the partial derivatives $\frac{\partial^{r} f}{\partial x_{i_{1}} \cdots \partial x_{i_{r}}}$ of $r$ th-order. It is clear that $J_{r}(f)$ is invariant if $K f$ is invariant, see $[1,8]$. (Convention: in this paper invariant means $G$-invariant.)

We shall denote by $S^{r}(V)$ the $r$-th symmetric power of $V$.

2.2. Yau's conjecture can be stated as follows: assume that $G=S L_{2}(\mathbb{C})$ and $V$ is a rational $G$-module, if $J(f)$ is invariant, then the set of highest weights of $J(f)$ is a subset of the set of highest weights of $A_{1}$.

2.3. Assume that $K$ is an algebraically closed field of characteristic 0 and $G$ is a connected semisimple algebraic group over $K$. Assume that $d$ is an integer greater than 2. Let $f \in A_{d}$ be such that $J(f)$ is invariant. Kempf showed that if $f=0$ is projectively smooth or $\rho$ is irreducible, then $f$ is an invariant; see [1]. Kempf also showed in the same paper that if $f$ is homogenous of degree greater than 2 and $J(f)$ is invariant, then there is an invariant homogeneous polynomial $g$ in $A$ with the same degree as of $f$ such that $J(f)=J(g)$.

\section{Main results}

In this section, we establish several module homomorphisms and show that Yau's conjecture is true for a connected semisimple algebraic group over an algebraically closed field of characteristic 0 (see Corollary 3.2).

Theorem 3.1. Keep the notations in 2.1. Let $e_{1}, \ldots, e_{n}$ be the standard basis of $V$ so that $x_{i}\left(e_{j}\right)=\delta_{i j}$.

(a) The linear map $A \otimes V \rightarrow A$ defined by $f \otimes e_{i} \rightarrow \frac{\partial f}{\partial x_{i}}$ is a homomorphism of $G$-modules. (Note that $G$ stands for a group or a Lie algebra.)

(b) The linear map $R \otimes V \rightarrow R$ defined by $f \otimes e_{i} \rightarrow \frac{\partial f}{\partial x_{i}}$ is a homomorphism of G-modules.

(c) Let $f \in R$. If $K f$ is a $G$-submodule of $R$, then $J(f)$ is a quotient module of $K f \otimes V$. In particular, if $f$ is invariant, then $J(f)$ is a quotient module of $V$.

(d) Assume that $f \in R$ is invariant, then $J_{r}(f)$ is a quotient module of the $r$-th symmetric power $S^{r}(V)$ of $V$.

Proof. Clearly (a) follows from (b). Let $f$ be a rational function in $R$.

(1) Assume that $G$ is a group. We need to show that the map sends $\tau(t) f \otimes$ $\rho(t)\left(e_{i}\right)$ to $\tau(t)\left(\frac{\partial f}{\partial x_{i}}\right)$ for any $t$ in $G$. Let $\rho(t)\left(e_{i}\right)=\sum_{j=1}^{n} a_{j i}(t) e_{j}$, then $\tau(t)\left(x_{k}\right)=$ $\sum_{j=1}^{n} a_{k j}\left(t^{-1}\right) x_{j}$. 
We first assume that $f=x_{1}^{a_{1}} \cdots x_{n}^{a_{n}}$ is a monomial. The linear map sends $\tau(t) f \otimes$ $\rho(t)\left(e_{i}\right)$ to

$$
\begin{aligned}
& \sum_{j=1}^{n} a_{j i}(t) \frac{\partial(\tau(t) f)}{\partial x_{j}} \\
& \quad=\sum_{j=1}^{n} a_{j i}(t) \sum_{k=1}^{n} a_{k} a_{k j}\left(t^{-1}\right) \tau(t)\left(x_{1}^{a_{1}} \cdots x_{k-1}^{a_{k-1}} x_{k}^{a_{k}-1} x_{k+1}^{a_{k+1}} \cdots x_{n}^{a_{n}}\right) \\
& \quad=\sum_{k=1}^{n} a_{k}\left(\sum_{j=1}^{n} a_{k j}\left(t^{-1}\right) a_{j i}(t)\right) \tau(t)\left(x_{1}^{a_{1}} \cdots x_{k-1}^{a_{k-1}} x_{k}^{a_{k}-1} x_{k+1}^{a_{k+1}} \cdots x_{n}^{a_{n}}\right) \\
& \quad=\sum_{k=1}^{n} \delta_{k i} a_{k} \tau(t)\left(x_{1}^{a_{1}} \cdots x_{k-1}^{a_{k-1}} x_{k}^{a_{k}-1} x_{k+1}^{a_{k+1}} \cdots x_{n}^{a_{n}}\right) \\
& \quad=a_{i} \tau(t)\left(x_{1}^{a_{1}} \cdots x_{i-1}^{a_{i-1}} x_{i}^{a_{i}-1} x_{i+1}^{a_{i+1}} \cdots x_{n}^{a_{n}}\right) \\
& =\tau(t)\left(\frac{\partial f}{\partial x_{i}}\right) .
\end{aligned}
$$

Hence, the linear map is a $G$-homomorphism from $A \otimes V$ to $A$ if $G$ is a group.

Now assume that $g, h$ are polynomial functions on $V$ and $f=h g^{-1}$. By the above computation, we have

$$
\sum_{j=1}^{n} a_{j i}(t) \frac{\partial(\tau(t) g)}{\partial x_{j}}=\tau(t)\left(\frac{\partial g}{\partial x_{i}}\right), \quad \sum_{j=1}^{n} a_{j i}(t) \frac{\partial(\tau(t) h)}{\partial x_{j}}=\tau(t)\left(\frac{\partial h}{\partial x_{i}}\right) .
$$

Therefore, the linear map sends $\tau(t)\left(h g^{-1}\right) \otimes \rho(t)\left(e_{i}\right)$ to

$$
\begin{aligned}
& \sum_{j=1}^{n} a_{j i}(t) \frac{\partial\left(\tau(t)\left(h g^{-1}\right)\right)}{\partial x_{j}} \\
& \quad=\sum_{j=1}^{n} a_{j i}(t) \frac{1}{\tau(t)\left(g^{2}\right)}\left[\tau(t)(g) \frac{\partial(\tau(t) h)}{\partial x_{j}}-\tau(t)(h) \frac{\partial(\tau(t) g)}{\partial x_{j}}\right] \\
& \quad=\frac{1}{\tau(t)\left(g^{2}\right)}\left[\tau(t)(g) \tau(t)\left(\frac{\partial h}{\partial x_{i}}\right)-\tau(t)(h) \tau(t)\left(\frac{\partial g}{\partial x_{i}}\right)\right] \\
& \quad=\tau(t)\left(\frac{\partial\left(h g^{-1}\right)}{\partial x_{i}}\right) .
\end{aligned}
$$

Hence, the linear map is a $G$-homomorphism from $R \otimes V$ to $R$ if $G$ is a group.

(2) Assume that $G$ is a Lie algebra. We need to show that the map sends $\tau(t) f \otimes$ $e_{i}+f \otimes \rho(t)\left(e_{i}\right)$ to $\tau(t)\left(\frac{\partial f}{\partial x_{i}}\right)$ for any $t$ in $G$. Let $\rho(t)\left(e_{i}\right)=\sum_{j=1}^{n} a_{j i} e_{j}$, then $\tau(t)\left(x_{j}\right)=$ $-\sum_{k=1}^{n} a_{j k} x_{k}$.

We first assume that $f=x_{1}^{a_{1}} \cdots x_{n}^{a_{n}}$ is a monomial. Set $b_{k}=a_{k}$, if $k \neq i, j$ and $b_{k}=a_{k}-1$ if $k=i, j$. Noting that

$$
\tau(t) f=\sum_{j=1}^{n}\left[a_{j} x_{1}^{a_{1}} \cdots x_{j-1}^{a_{j-1}} x_{j}^{a_{j}-1} x_{j+1}^{a_{j+1}} \cdots x_{n}^{a_{n}}\left(-\sum_{k=1}^{n} a_{j k} x_{k}\right)\right],
$$


we see that $\tau(t) f \otimes e_{i}+f \otimes \rho(t)\left(e_{i}\right)$ is sent to

$$
\begin{aligned}
\sum_{j \neq i}^{n}[ & \left.a_{j} a_{i} x_{1}^{b_{1}} x_{2}^{b_{2}} \cdots x_{n}^{b_{n}}\left(-\sum_{k=1}^{n} a_{j k} x_{k}\right)\right] \\
& +a_{i}\left(a_{i}-1\right) x_{1}^{a_{1}} \cdots x_{i-1}^{a_{i-1}} x_{i}^{a_{i}-2} x_{i+1}^{a_{i+1}} \cdots x_{n}^{a_{n}}\left(-\sum_{k=1}^{n} a_{i k} x_{k}\right) \\
& +\sum_{j=1}^{n} a_{j}\left(-a_{j i}\right) x_{1}^{a_{1}} \cdots x_{j-1}^{a_{j-1}} x_{j}^{a_{j}-1} x_{j+1}^{a_{j+1}} \cdots x_{n}^{a_{n}} \\
& +\sum_{j=1}^{n} a_{j} a_{j i} x_{1}^{a_{1}} \cdots x_{j-1}^{a_{j-1}} x_{j}^{a_{j}-1} x_{j+1}^{a_{j+1}} \cdots x_{n}^{a_{n}} \\
= & \sum_{j \neq i}^{n}\left[a_{j} a_{i} x_{1}^{b_{1}} x_{2}^{b_{2}} \cdots x_{n}^{b_{n}}\left(-\sum_{k=1}^{n} a_{j k} x_{k}\right)\right] \\
& +a_{i}\left(a_{i}-1\right) x_{1}^{a_{1}} \cdots x_{i-1}^{a_{i-1}} x_{i}^{a_{i}-2} x_{i+1}^{a_{i+1}} \cdots x_{n}^{a_{n}}\left(-\sum_{k=1}^{n} a_{i k} x_{k}\right) \\
= & \tau(t)\left(\frac{\partial f}{\partial x_{i}}\right) .
\end{aligned}
$$

So the linear map is a $G$-homomorphism of $G$-module from $A \otimes V$ to $A$ if $G$ is a Lie algebra.

Now assume that $g, h$ are polynomial functions on $V$ and $f=h g^{-1}$. By the above computation, we have

$$
\frac{\partial(\tau(t) g)}{\partial x_{i}}+\sum_{j=1}^{n} a_{j i} \frac{\partial g}{\partial x_{j}}=\tau(t)\left(\frac{\partial g}{\partial x_{i}}\right), \quad \frac{\partial(\tau(t) h)}{\partial x_{i}}+\sum_{j=1}^{n} a_{j i} \frac{\partial h}{\partial x_{j}}=\tau(t)\left(\frac{\partial h}{\partial x_{i}}\right) .
$$

Noting that $\tau(t)\left(g^{-a}\right)=-a g^{-a-1} \tau(t)(g)$ for $a \geq 1$ and $\tau(t)\left(h g^{-1}\right)=g^{-1} \tau(t)(h)+$ $h \tau(t)\left(g^{-1}\right)$, we see that $\tau(t)\left(h g^{-1}\right) \otimes e_{i}+h g^{-1} \otimes \rho(t)\left(e_{i}\right)$ is sent to

$$
\begin{aligned}
& \frac{\partial\left(g^{-1} \tau(t)(h)\right)}{\partial x_{i}}-\frac{\partial\left(g^{-2} h \tau(t)(g)\right)}{\partial x_{i}}+\sum_{j=1}^{n} a_{j i} \frac{\partial\left(g^{-1} h\right)}{\partial x_{j}} \\
&=g^{-1} \frac{\partial(\tau(t)(h))}{\partial x_{i}}-g^{-2} \tau(t)(h) \frac{\partial g}{\partial x_{i}}+2 g^{-3} h \tau(t)(g) \frac{\partial g}{\partial x_{i}} \\
& \quad-g^{-2} \tau(t)(g) \frac{\partial h}{\partial x_{i}}-g^{-2} h \frac{\partial(\tau(t)(g))}{\partial x_{i}}+\sum_{j=1}^{n} a_{j i}\left(g^{-1} \frac{\partial h}{\partial x_{j}}-g^{-2} h \frac{\partial g}{\partial x_{i}}\right) \\
&=g^{-1} \tau(t)\left(\frac{\partial h}{\partial x_{i}}\right)-g^{-2} \tau(t)(h) \frac{\partial g}{\partial x_{i}}+2 g^{-3} h \tau(t)(g) \frac{\partial g}{\partial x_{i}} \\
& \quad-g^{-2} \tau(t)(g) \frac{\partial h}{\partial x_{i}}-g^{-2} h \tau(t)\left(\frac{\partial g}{\partial x_{i}}\right) \\
&= \tau(t)\left(\frac{\partial\left(h g^{-1}\right)}{\partial x_{i}}\right) .
\end{aligned}
$$

Hence, the linear map is a $G$-homomorphism from $R \otimes V$ to $R$ if $G$ is a Lie algebra. 
(c) follows from (b) and (d) follows from (c). The theorem is proved.

Corollary 3.2. Assume that $K$ is an algebraically closed field of characteristic 0 and $G$ is a connected semisimple algebraic group over $K$. If $f \in A$ is homogenous of degree greater than 2 and $J(f)$ is invariant, then $J_{r}(f)$ is a quotient module of $S^{r}(V)$.

Proof. According [1, Theorem 13], there is an invariant homogenous polynomial $g$ in $A$ with the same degree as of $f$ such that $J(f)=J(g)$, the corollary then follows from Theorem $3.1(\mathrm{~d})$.

Remark. Since $A_{1}$ is the dual module of $V$, so that for $G=S L_{2}(K), V$ is isomorphic $A_{1}$ as $G$-modules, Yau's conjecture then follows from Corollary 3.2 for $G=$ $S L_{2}(\mathbb{C})$ and $r=1$. Corollary 3.2 shows that Yau's conjecture holds in a more general setting.

Theorem 3.3. Keep the notations in 2.1.

(a) The linear map $A \rightarrow A_{1} \otimes A$ defined by $f \rightarrow \sum_{i=1}^{n} x_{i} \otimes \frac{\partial f}{\partial x_{i}}$ is a homomorphism of $G$-modules.

(b) The linear map $R \rightarrow A_{1} \otimes R$ defined by $f \rightarrow \sum_{i=1}^{n} x_{i} \otimes \frac{\partial f}{\partial x_{i}}$ is a homomorphism of $G$-modules.

Proof. Assume that $f$ is a rational function in $R$.

(1) Let $G$ be a group and $t \in G$. We need to show that the map sends $\tau(t) f$ to $\sum_{i=1}^{n} \tau(t) x_{i} \otimes \tau(t)\left(\frac{\partial f}{\partial x_{i}}\right)$. Let $\tau(t)\left(x_{i}\right)=\sum_{j=1}^{n} b_{j i} x_{j}$.

First we assume that $f=x_{1}^{a_{1}} \cdots x_{n}^{a_{n}}$ is a monomial. The linear map sends $\tau(t) f$ to

$$
\begin{aligned}
& \sum_{j=1}^{n} x_{j} \otimes \frac{\partial(\tau(t) f)}{\partial x_{j}} \\
& \quad=\sum_{j=1}^{n} x_{j} \otimes \sum_{i=1}^{n} a_{i} b_{j i} \tau(t)\left(x_{1}^{a_{1}} \cdots x_{i-1}^{a_{i-1}} x_{i}^{a_{i}-1} x_{i+1}^{a_{i+1}} \cdots x_{n}^{a_{n}}\right) \\
& \quad=\sum_{i=1}^{n}\left[\sum_{j=1}^{n} b_{j i} x_{j} \otimes a_{i} \tau(t)\left(x_{1}^{a_{1}} \cdots x_{i-1}^{a_{i-1}} x_{i}^{a_{i}-1} x_{i+1}^{a_{i+1}} \cdots x_{n}^{a_{n}}\right)\right] \\
& \quad=\sum_{i=1}^{n} \tau(t) x_{i} \otimes \tau(t)\left(\frac{\partial f}{\partial x_{i}}\right) .
\end{aligned}
$$

Hence, the linear map is a $G$-homomorphism from $A$ to $A_{1} \otimes A$ if $G$ is a group.

Now assume that $g, h$ are polynomial functions on $V$ and $f=h g^{-1}$. By the above computation, we have

$$
\begin{aligned}
& \sum_{i=1}^{n} x_{i} \otimes \frac{\partial(\tau(t) g)}{\partial x_{i}}=\sum_{i=1}^{n} \tau(t) x_{i} \otimes \tau(t)\left(\frac{\partial g}{\partial x_{i}}\right) \\
& \sum_{i=1}^{n} x_{i} \otimes \frac{\partial(\tau(t) h)}{\partial x_{i}}=\sum_{i=1}^{n} \tau(t) x_{i} \otimes \tau(t)\left(\frac{\partial h}{\partial x_{i}}\right) .
\end{aligned}
$$


Therefore, the linear map sends $\tau(t)\left(h g^{-1}\right)$ to

$$
\begin{aligned}
& \sum_{i=1}^{n} x_{i} \otimes \frac{\partial\left(\tau(t)\left(h g^{-1}\right)\right.}{\partial x_{i}} \\
& \quad=\sum_{i=1}^{n} x_{i} \otimes\left[\tau(t)\left(g^{-1}\right) \frac{\partial(\tau(t) h)}{\partial x_{i}}-\tau\left(h g^{-2}\right) \frac{\partial(\tau(t) g)}{\partial x_{i}}\right] \\
& \quad=\sum_{i=1}^{n} \tau(t) x_{i} \otimes\left[\tau(t)\left(g^{-1}\right) \tau(t)\left(\frac{\partial h}{\partial x_{i}}\right)-\tau\left(h g^{-2}\right) \tau(t)\left(\frac{\partial g}{\partial x_{i}}\right)\right] \\
& \quad=\sum_{i=1}^{n} \tau(t) x_{i} \otimes \tau(t) \frac{\partial\left(h g^{-1}\right)}{\partial x_{i}} .
\end{aligned}
$$

Hence, the linear map is a $G$-homomorphism from $R$ to $A_{1} \otimes R$ if $G$ is a group.

(2) Let $G$ be a Lie algebra. We need show that the map sends $\tau(t) f$ to $\sum_{i=1}^{n}\left[x_{i} \otimes\right.$ $\left.\tau(t)\left(\frac{\partial f}{\partial x_{i}}\right)+\tau(t) x_{i} \otimes \frac{\partial f}{\partial x_{i}}\right]$. Let $\tau(t)\left(x_{i}\right)=\sum_{j=1}^{n} b_{j i} x_{j}$.

First we assume that $f=x_{1}^{a_{1}} \cdots x_{n}^{a_{n}}$ is a monomial. We have

$$
\tau(t) f=\sum_{j=1}^{n}\left(a_{j} x_{1}^{a_{1}} \cdots x_{j-1}^{a_{j-1}} x_{j}^{a_{j}-1} x_{j+1}^{a_{j+1}} \cdots x_{n}^{a_{n}} \sum_{k=1}^{n} b_{k j} x_{k}\right) .
$$

Let $b_{r}=a_{r}$ if $r \neq i, j$ and $b_{r}=a_{r}-1$ if $r=i, j$. Then the linear map sends $\tau(t) f$ to

$$
\begin{aligned}
\sum_{i=1}^{n} & x_{i} \otimes \frac{\partial(\tau(t) f)}{\partial x_{i}} \\
= & \sum_{i=1}^{n} x_{i} \otimes \sum_{j \neq i}^{n}\left(a_{j} a_{i} x_{1}^{b_{1}} x_{2}^{b_{2}} \cdots x_{n}^{b_{n}} \sum_{k=1}^{n} b_{k j} x_{k}\right) \\
& +\sum_{i=1}^{n} x_{i} \otimes a_{i}\left(a_{i}-1\right) x_{1}^{a_{1}} \cdots x_{i-1}^{a_{i-1}} x_{i}^{a_{i}-2} x_{i+1}^{a_{i+1}} \cdots x_{n}^{a_{n}}\left(\sum_{k=1}^{n} b_{k i} x_{k}\right) \\
& +\sum_{i=1}^{n} x_{i} \otimes \sum_{j=1}^{n} a_{j} b_{i j} x_{1}^{a_{1}} \cdots x_{j-1}^{a_{j-1}} x_{j}^{a_{j}-1} x_{j+1}^{a_{j+1}} \cdots x_{n}^{a_{n}} \\
= & \sum_{i=1}^{n} x_{i} \otimes \tau(t)\left(\frac{\partial f}{\partial x_{i}}\right)+\sum_{j=1}^{n}\left(\sum_{i=1}^{n} b_{i j} x_{i}\right) \otimes a_{j} x_{1}^{a_{1}} \cdots x_{j-1}^{a_{j-1}} x_{j}^{a_{j}-1} x_{j+1}^{a_{j+1}} \cdots x_{n}^{a_{n}} \\
= & \sum_{i=1}^{n} x_{i} \otimes \tau(t)\left(\frac{\partial f}{\partial x_{i}}\right)+\sum_{j=1}^{n} \tau(t) x_{j} \otimes \frac{\partial f}{\partial x_{j}} \\
= & \sum_{i=1}^{n}\left[x_{i} \otimes \tau(t)\left(\frac{\partial f}{\partial x_{i}}\right)+\tau(t) x_{i} \otimes \frac{\partial f}{\partial x_{i}}\right]
\end{aligned}
$$

Hence, the linear map is a $G$-homomorphism from $A$ to $A_{1} \otimes A$ if $G$ is a Lie algebra. 
Now assume that $g, h$ are polynomial functions on $V$ and $f=h g^{-1}$. By the above computation, we have

$$
\begin{aligned}
& \sum_{i=1}^{n} x_{i} \otimes \frac{\partial(\tau(t) g)}{\partial x_{i}}=\sum_{i=1}^{n}\left[x_{i} \otimes \tau(t)\left(\frac{\partial g}{\partial x_{i}}\right)+\tau(t) x_{i} \otimes \frac{\partial g}{\partial x_{i}}\right], \\
& \sum_{i=1}^{n} x_{i} \otimes \frac{\partial(\tau(t) h)}{\partial x_{i}}=\sum_{i=1}^{n}\left[x_{i} \otimes \tau(t)\left(\frac{\partial h}{\partial x_{i}}\right)+\tau(t) x_{i} \otimes \frac{\partial h}{\partial x_{i}}\right] .
\end{aligned}
$$

Therefore, the linear map sends $\tau(t)\left(h g^{-1}\right)=g^{-1} \tau(t)(h)-g^{-2} h \tau(t)(g)$ to

$$
\begin{aligned}
\sum_{i=1}^{n} & x_{i} \otimes\left[\frac{\partial\left(g^{-1} \tau(t) h\right)}{\partial x_{i}}-\frac{\partial\left(g^{-2} h \tau(t) g\right)}{\partial x_{i}}\right] \\
= & \sum_{i=1}^{n} x_{i} \otimes\left[g^{-1} \frac{\partial(\tau(t) h)}{\partial x_{i}}-g^{-2} \tau(t)(h) \frac{\partial g}{\partial x_{i}}\right. \\
& \left.+2 g^{-3} h \tau(t)(g) \frac{\partial g}{\partial x_{i}}-g^{-2} \tau(t)(g) \frac{\partial h}{\partial x_{i}}-g^{-2} h \frac{\partial(\tau(t) g)}{\partial x_{i}}\right] \\
= & \sum_{i=1}^{n} x_{i} \otimes\left[g^{-1} \tau(t)\left(\frac{\partial h}{\partial x_{i}}\right)-g^{-2} \tau(t)(h) \frac{\partial g}{\partial x_{i}}\right. \\
& \left.+2 g^{-3} h \tau(t)(g) \frac{\partial g}{\partial x_{i}}-g^{-2} \tau(t)(g) \frac{\partial h}{\partial x_{i}}-g^{-2} h \tau(t)\left(\frac{\partial g}{\partial x_{i}}\right)\right] \\
& +\sum_{i=1}^{n} \tau(t) x_{i} \otimes\left(g^{-1} \frac{\partial h}{\partial x_{i}}-g^{-2} h \frac{\partial g}{\partial x_{i}}\right) \\
= & \sum_{i=1}^{n}\left[x_{i} \otimes \tau(t)\left(\frac{\partial\left(h g^{-1}\right)}{\partial x_{i}}\right)+\tau(t) x_{i} \otimes \frac{\partial\left(h g^{-1}\right)}{\partial x_{i}}\right] .
\end{aligned}
$$

Hence, the linear map is a $G$-homomorphism from $R$ to $A_{1} \otimes R$ if $G$ is a Lie algebra.

The theorem is proved.

Corollary 3.4. Keep the notations in 2.1.

(a) If $f \in R$ is invariant, then $\sum_{i=1}^{n} x_{i} \otimes \frac{\partial f}{\partial x_{i}}$ is invariant in $A_{1} \otimes R$.

(b) If $d$ is a positive integer and $d$ is invertible in $K$, then $A_{d}$ is a direct summand of $A_{1} \otimes A_{d-1}$.

Proof. (a) is clear from Theorem 3.3 (b). We have a natural $G$-homomorphism $\psi$ : $A_{1} \otimes R \rightarrow R, x_{i} \otimes f \rightarrow x_{i} f$. Let $\varphi$ be the map in Theorem 3.3 (b) and $f \in A_{d}$. Then $\psi \varphi(f)=d f$. Since $d$ is invertible in $K$, we have $A_{1} \otimes A_{d-1} \simeq A_{d} \oplus \operatorname{ker} \psi^{\prime}$, here $\psi^{\prime}$ is the restriction of $\psi$ to $A_{1} \otimes A_{d-1}$. The corollary is proved.

3.5. Question Keep the notations in 2.1. Let $f \in R$ be such that $J(f)$ is invariant. (a) describe the module structure of $J(f)$, (b) does there exist $g \in R$ such that $K g$ is invariant and $J(g)=J(f)$ ? (c) describe the set $\{g \in R \mid J(g)=J(f)\}$. 


\section{A direct proof for Yau's conjecture}

4.1. We use the setup of [8] (see also $[4,10,11]$ ) to give a direct proof for Yau's conjecture, see part (c) of Proposition 4.2. Let $K$ be an algebraically closed field of characteristic 0 and $A$ be as in 2.1. Let $H, X, Y$ be the standard basis of $s l_{2}(K)$ so we have $[X, Y]=H,[H, X]=2 X,[H, Y]=-2 Y$.

Let $l_{1}, \ldots, l_{r}$ be positive integers such that their sum is $n$. For $1 \leq i \leq r$, define

$$
\begin{aligned}
H_{i}= & \left(l_{i}-1\right) x_{l_{1}+\cdots+l_{i-1}+1} \frac{\partial}{\partial x_{l_{1}+\cdots+l_{i-1}+1}} \\
& +\cdots+\left(l_{i}-2 j+1\right) x_{l_{1}+\cdots+l_{i-1}+j} \frac{\partial}{\partial x_{l_{1}+\cdots+l_{i-1}+j}} \\
& +\cdots+\left(-l_{i}+1\right) x_{l_{1}+\cdots+l_{i}} \frac{\partial}{\partial x_{l_{1}+\cdots+l_{i}}}, \\
X_{i}= & \left(l_{i}-1\right) x_{l_{1}+\cdots+l_{i-1}+1} \frac{\partial}{\partial x_{l_{1}+\cdots+l_{i-1}+2}} \\
& +\cdots+j\left(l_{i}-j\right) x_{l_{1}+\cdots+l_{i-1}+j} \frac{\partial x_{l_{1}+\cdots+l_{i-1}+j+1}}{\partial} \\
& +\cdots+\left(l_{i}-1\right) x_{l_{1}+\cdots+l_{i}-1} \frac{\partial x_{l_{1}+\cdots+l_{i}}}{\partial} \\
Y_{i}= & x_{l_{1}+\cdots+l_{i-1}+2} \frac{\partial x_{l_{1}+\cdots+l_{i-1}+1}}{\partial x_{l_{1}+\cdots+l_{i-1}+j-1}} \\
& +\cdots+x_{l_{1}+\cdots+l_{i-1}+j} \\
& +\cdots+x_{l_{1}+\cdots+l_{i}} \frac{\partial}{\partial x_{l_{1}+\cdots+l_{i}-1}} .
\end{aligned}
$$

Then the map $H \rightarrow H_{1}+\cdots+H_{r}, X \rightarrow X_{1}+\cdots+X_{r}$ and $Y \rightarrow Y_{1}+\cdots+Y_{r}$ defines an action of $s l_{2}(K)$ on $A$. Under this action, $x_{l_{1}+\cdots+l_{i-1}+j}$ is a vector of weight $l_{i}-2 j+1$, and the elements $x_{l_{1}+\cdots+l_{i-1}+1}, x_{l_{1}+\cdots+l_{i-1}+2}, \ldots, x_{l_{1}+\cdots+l_{i-1}+l_{i}-1}, x_{l_{1}+\cdots+l_{i}}$ span an irreducible submodule of $A$ of dimension $l_{i}$.

Proposition 4.1. $\quad$ (a) The linear map $A \otimes A_{1} \rightarrow A, f \otimes x_{i} \rightarrow(-1)^{i} \frac{\partial f}{\partial x_{i^{\prime}}}$ is a homomorphism of $\operatorname{sl}_{2}(K)$-modules, $i^{\prime}$ is determined by the following conditions: (1) $x_{i^{\prime}}$ is in the submodule generated by $x_{i}$ and (2) the weight of $x_{i^{\prime}}$ is opposite to that of $x_{i}$, i.e., $i^{\prime}=l_{1}+\cdots+l_{i-1}+l_{i}-j+1$ if $i=l_{1}+\cdots+l_{i-1}+j$ for $1 \leq j \leq l_{i}$.

(b) If $f \in A$ is invariant, then $J(f)$ is a quotient module of $A_{1}$.

(c) Let $f \in A_{d}$ and $d \geq 3$. If $J(f)$ is invariant, then $J(f)$ is a quotient module of $A_{1}$. In particular, in this case the set of highest weights of $J(f)$ is a subset of the set of highest weights of $A_{1}$.

Proof. Part (a) can be checked by a direct calculation. Part (b) follows from (a). Now we prove part (c). By [1, Theorem 13], there exists $g \in A_{d}$ such that $g$ is invariant and $J(g)=J(f)$. Thus, (c) follows from (b). The proposition is proved.

Remark. It is easy to see that the homomorphism in Proposition 4.2 (a) can be uniquely extended to homomorphism $R \otimes A_{1} \rightarrow R$. 


\section{Acknowledgments}

I thank Stephen Yau for talking me his interesting conjecture during a lunch and for explaining me the background of his conjecture. Supported in part by the President Foundation of the AMSS, Chinese Academy of Sciences.

\section{References}

[1] G. R. Kempf. Jacobians and invariants, Invent. Math. 112 (1993), 315-321.

[2] J. N. Mather and S. S.-T. Yau. Classification of isolated hypersurface singularities by their moduli algebras, Invent. Math. 69 (1982), 243-251.

[3] C. Seeley and S. S.-T. Yau. Variation of complex structures and variation of Lie algebras, Invent. Math. 79 (1990), 545-565.

[4] J. Sampson, S. S.-T. Yau, and Y. Yu. Classification of gradient space as sl(2, C)-module I, Amer. J. Math, 114 (1992), 1147-1161.

[5] S. S.-T. Yau. Continuous family of finite-dimensional representations of a solvable Lie algebra arising from singularities, Proc. Natl Acad. Sci. USA, 80(24) (1983), 7694-7696.

[6] S. S.-T. Yau. Solvable Lie algebras and generalized Cartan matrices arising from isolated singularities, Math. Z. 191 (1986), 489-506.

[7] S. S.-T. Yau. Singularities defined by sl(2, C) invariant polynomials and solvability of Lie algebras arising from isolated singularities, Amer. J. Math., 108(5) (1986), 1215-1239.

[8] S. S.-T. Yau. Classification of Jacobian ideals invariant by sl(2, C) actions, Mem. Amer. Math. Soc., 72 (1988), pp. 180.

[9] S. S.-T. Yau. Solvability of Lie algebras arising from isolated singularities and nonisolatedness of singularities defined by sl(2, C) invariant polynomials, Amer. J. Math., 113(5) (1991), 773-778.

[10] S. S.-T. Yau, Y. Yu, and HuaiQing Zuo. Classification of gradient space of dimension 8 by a reducible sl(2,C) action, Science China A: Math., 52 (2009), 2792-2828.

[11] Y. Yu. On Jacobian ideals inviariant by a reducible sl $(2, \mathbf{C})$ action, Trans. Amer. Math. Soc., 348(7) (1996), 2759-2791.

Hua Loo-Keng Key Laboratory of Mathematics and Institute of Mathematics

Chinese ACADEMy OF SCIEnCES

BEIJING 100190

PeOple's Republic of ChinA

E-mail address: nanhua@math.ac.cn 
